\title{
Incidence of midportion Achilles tendinopathy in the general population
}

\author{
S de Jonge, ${ }^{1,2} \mathrm{C}$ van den Berg, ${ }^{1} \mathrm{R} \mathrm{J}$ de Vos, ${ }^{1} \mathrm{H} \mathrm{J} \mathrm{L}$ van der Heide, ${ }^{3} \mathrm{~A}$ Weir, \\ J A N Verhaar, ${ }^{2}$ S M A Bierma-Zeinstra, ${ }^{2,4} \mathrm{~J} \mathrm{~L} \mathrm{Tol}^{1}$
}

${ }^{1}$ Sports Medicine Department The Hague Medical Centre, Leidschendam,

The Netherlands ${ }^{2}$ Department of Orthopaedics, Erasmus Medical Centre, Rotterdam, The Netherlands ${ }^{3}$ Department of Orthopaedics, Leiden University Medical Centre, Leiden,

The Netherlands

${ }^{4}$ Department of General Practice, Erasmus University Medical Centre, Rotterdam, The Netherlands

\section{Correspondence to} $S$ de Jonge, Orthopaedic Research Laboratory, Erasmus MC, PO Box 2040, 3000 CA Rotterdam, The Netherlands: s.dejonge@erasmusmc.nl

Accepted 3 August 2011

\section{ABSTRACT}

Background Achilles tendon disorders, like Achilles tendinopathy, are very common among athletes. In the general population, however, knowledge about the incidence of Achilles tendinopathy is lacking.

Design Cross-sectional study.

Methods In a cohort of 57.725 persons registered in primary care, the number of patients visiting the general practitioner (GP) with diagnosis of mid-portion Achilles tendon problems was counted using computerised registration networks of GPs in 2009. Subsequently, the authors assessed associations of these rates with demographic characteristics.

Results The incidence rate of Achilles tendinopathy is 1.85 per 1,000 Dutch GP registered patients. In the adult population (21-60 years), the incidence rate is 2.35 per 1,000 . In $35 \%$ of the cases, a relationship with sports activity was recorded.

Conclusion This is the first report on incidence rates of mid-portion Achilles tendinopathy in general practice. With an incidence of 1.85 per 1,000 registered persons, Achilles tendinopathy is frequently seen by GPs. The actual incidence might even be higher due to study limitations. More research on the frequency of this injury is required.

\section{INTRODUCTION}

Achilles tendon problems frequently occur in athletes and are supposed to be common as well in the general population. The frequency of Achilles tendinopathy in athletes has been reported in several studies. Elite long-distance runners have a lifetime risk of $52 \%$, and the lifetime incidence of 416 participants of Finnish conscription was $5.9 \% .{ }^{1}$ Others reported annual incidence rates of Achilles tendon disorders of $7 \%$ and $9 \%$, respectively, in top-level runners. ${ }^{23}$ Among the military population, an incidence of 2.98 per 1,000 person years was found. ${ }^{4}$ A cohort study of 725 male marathon runners reported an incidence of $7.4 \%$ in the month before the Rotterdam marathon. ${ }^{5}$

While these frequency rates were recorded in the sporting population, Achilles tendinopathy is not always associated with excessive physical activity; it is also seen in patients who do not participate in sports. ${ }^{6-8}$ To our knowledge, there are no studies on the incidence of Achilles tendinopathy in the general population. Incidence rates are useful for studying trends in occurrence of diseases, future intervention studies, and for burden of disease estimates.

To obtain this incidence rate, a cross-sectional study within the Dutch general practice setting was performed. In the Netherlands, every non-institutionalised inhabitant is registered with a general practitioner. In case of a health problem, the general practice is intended to be a gatekeeper to specialist care. The primary aim of this study was to ascertain the frequency of mid-portion Achilles tendinopathy seen in the general practitioner (GP) setting.

\section{METHODS}

In this cross-sectional study, data were obtained from computerised registration systems of Dutch GPs. General practices were approached for participation by mail and telephone. After permission, the researcher visited the practices.

During this visit, all electronic patient files for consultations in 2009 were systematically searched under supervision of the GP. GPs in the Netherlands use International Classification of Primary Care (ICPC) ${ }^{9}$ codes to register the reason for the visit. These codes include disease-specific codes as well as complaint specific codes. There is, however, no specific code for Achilles tendinopathy. Another sensitive way of searching for certain diagnosis is to use diagnosis specific words in the free text of the records. We searched for the term achil to find as many records describing the diagnosis of Achilles tendinopathy (eg, terms as Achillodynia could also be found). Medical files of the found records were read by a single researcher (CvdB) to assess whether they met the inclusion criteria. For the diagnosis of Achilles tendinopathy, a description of pain in the Achilles tendon above the insertion was required. Lack of this description resulted in exclusion. When the first contact with the GP for complaints was in the year 2009 without a previous visit in 2008 , the patient was scored as an incident case. In addition, age, gender, date of presentation and sports activity (related to complaints) were recorded if these had been recorded in the medical record. The medical record was also screened for diabetes mellitus (DM) type 1 and 2 .

Statistical analysis was performed using SPSS (version 17). Incidence rates were calculated separately for age group and sex.

\section{RESULTS}

Between February 2011 and April 2011, 128 general practices were approached. Fourteen practitioners did not answer repeated phone calls and 94 GPs refused to participate. Twenty GPs at different geographic locations responded positively to the invitation and were visited by the researcher. 
Table 1 Age and sex specific incidence rates for Achilles tendinopathy in 20 general practices



$\mathrm{n}=$ number of persons with Achilles tendinopathy; Persons = number of registered persons; IR, incidence rate $=$ number cases per 1000 registered persons.

The 20 participating practices contained 57.725 registered persons, with an average of 2,886 persons in each practice (range $1,757-6,486)$.

The research strategy "Achil" in the free text resulted in 277 cases in 2009 . After screening the medical files, 116 cases of mid-portion Achilles tendinopathy could be recorded. Frequently reported other Achilles tendon disorders were insertional disorder, lash and bursitis. The prevalence rate of Achilles tendinopathy is 2.01 per 1,000 registered patients.

Of these 116 prevalent cases, 9 patients had visited the general practitioner in 2008 for the same reason, resulting in 107 incident cases in 2009. The incidence rate for mid-portion Achilles tendinopathy is 1.85 per 1,000 registered patients.

The 107 incident cases contained 56 females (52.3\%) and 51 males (47.7\%). Age- and sex-specific incidence rates are given in table 1 . The overall incidence rate in the adult population between 21 and 60 years is 2.35 per 1,000. The mean age at time of presentation within the cases was 43.4 years (range 7-85 years). Mid-portion Achilles tendinopathy equally affects women and men.

Mean duration of symptoms at presentation (reported in 39 cases) was 11.3 weeks (range 1-52). In 37 cases (34.6\%), a relationship with sports activity was recorded. Ten patients $(9.3 \%)$ were known to have DM-two patients with type $1 \mathrm{DM}$ and eight patients with type 2 DM. Table 2 shows the patient characteristics of the case group compared with the study population and the Dutch population.

\section{DISCUSSION}

This is the first study presenting incidence rates of Achilles tendinopathy in the general practice. The overall incidence rate is 1.85 per 1,000 registered persons per year, and for the adult population it is 2.35. There are no other studies on the incidence rates of Achilles tendinopathy in the general population to compare these results with. The prevalence rate was higher than that for another tendinopathy like lateral epicondylitis of
$1.3 \%$ (men) and $1.1 \%$ (women) in 9,696 persons registered at two general practitioners. ${ }^{10}$

The incidence rates in our study are lower than the incidence of 2.98 found in the military population ${ }^{4}$ and than the annual incidence $7 \%$ and $9 \%$ in top-level runners. ${ }^{23}$

While it seems plausible that the incidence rate of Achilles tendon injuries is higher in athletes than within a more general population, only in $35 \%$ of the cases in our study was a relationship with sports activity described. Intrinsic risk factors such as body weight and insulin resistance might also play a role. ${ }^{11}$ Although we could not assess information on body weight, the diagnosis of DM was available from the medical records. DM seemed more prevalent among the incident cases compared with the general Dutch population (table 2). However, more research is needed to draw any conclusions on a causative association The minor difference between the incidence and prevalence rate suggests that most patients only visit their GP once with their Achilles tendon problems. This might be because of a short duration of symptoms or that the patients go to a physiotherapist or sports physician afterwards.

This cross-sectional study has some limitations, due to which the actual incidence of Achilles tendinopathy in the general population is probably higher than observed in this study.

Firstly, not every person with a certain complaint will visit a healthcare professional. In a cross-sectional population-based study, Picavet et al reported that approximately $50 \%$ of the patients with musculoskeletal complaints visited a healthcare professional. ${ }^{12}$ However, those with severe or persistent complaints will probably seek healthcare.

Second, while most patients in the Dutch healthcare system will visit their GP before being referred to a therapist or specialist, since 2006 patients can visit a physiotherapist or sports physician without referral by a GP. It would be interesting to know the incidence rates of Achilles tendinopathy within the patient population of the Dutch physiotherapists and sports physicians

Table 2 Patient characteristics in the cases with Achilles tendinopathy, in the total study population, and in the Dutch population (as recorded by CBS, Statistics Netherlands http://www.cbs.nl 2009)

\begin{tabular}{llll}
\hline & $\begin{array}{l}\text { Patients with Achilles } \\
\text { tendinopathy }(\mathbf{9 5} \% \mathbf{C l}) \mathbf{n}=\mathbf{2 0 7}\end{array}$ & Study population $\mathbf{n = 5 7 . 7 2 5}$ & $\begin{array}{l}\text { Dutch population in year 2009 } \\
\mathbf{n = 1 6 . 4 8 5 . 7 8 7}\end{array}$ \\
\hline Gender & & & \\
Male & $47.7 \%(95 \% \mathrm{Cl}, 38.0$ to 57.3$)$ & $48.1 \%$ & $49.5 \%$ \\
$\quad$ Female & $52.3 \%(95 \% \mathrm{Cl}, 42.7$ to 62.0$)$ & $51.9 \%$ & $50.5 \%$ \\
Diabetes mellitus & $9.3 \%(95 \% \mathrm{Cl}, 3.7$ to 15.0$)$ & & $4.1 \%$ \\
$\quad$ Type 1 & $1.8 \%(95 \% \mathrm{Cl}, 0$ to 4.5$)$ & & $0.8 \%$ \\
Type 2 & $7.5 \%(95 \% \mathrm{Cl}, 2.4$ to 12.5$)$ & & $3.3 \%$ \\
Age groups & & & \\
$<20$ years & $12.1 \%(95 \% \mathrm{Cl}, 5.9$ to 18.4$)$ & $22.0 \%$ & $23.9 \%$ \\
$21-40$ years & $31.8 \%(95 \% \mathrm{Cl}, 22.8$ to 40.7$)$ & $25.8 \%$ & $25.7 \%$ \\
$41-60$ years & $39.3 \%(95 \% \mathrm{Cl}, 29.8$ to 48.7$)$ & $30.2 \%$ & $35.5 \%$ \\
$>60$ years & $16.8 \%(95 \% \mathrm{Cl}, 9.6$ to 24.0$)$ & $22.0 \%$ & $15.0 \%$ \\
\hline
\end{tabular}


as well, but since the denominators of these populations are unknown, no exact incidence rates could be obtained.

Finally, because there is no specific registration code (ICPC) available for Achilles tendinopathy, GPs register this injury using other or more general codes. The search strategy "Achil" is considered to be highly sensitive for Achilles tendon problems; however, there is a possibility of missing some cases. Furthermore, the 13 patients less than 20 years old might have a growth-related disorder rather than mid-portion Achilles tendinopathy.

In concordance with other studies, ${ }^{413}$ most cases were encountered in the middle-aged population (age group 41-60 years). It is unclear why Achilles tendinopathy is more frequent within this age group. A possible explanation might be a higher incidence of degenerative tendons, susceptible to overuse, in the elderly.

\section{CONCLUSION}

In conclusion, this is the first study to report an incidence rate of Achilles tendinopathy in the general population. With an incidence of 1.85 per 1,000 registered patients, and of 2.35 in the adult population, more research on pathophysiology and therapy is warranted.

Acknowledgements The authors would like to thank Marienke van Middelkoop, PhD (Department of General Practice, Erasmus University Medical Centre, Rotterdam, the Netherlands), for her contribution to the study design. The authors thank the general practitioners for their participation in the study.
Competing interests None.

Provenance and peer review Not commissioned; externally peer reviewed.

\section{REFERENCES}

1. Kujala UM, Sarna S, Kaprio J. Cumulative incidence of achilles tendon rupture and tendinopathy in male former elite athletes. Clin J Sport Med 2005;15:133-5.

2. Johannsen FE, Gam AN. [Achillodynia is not just a sports injury]. Ugeskr Laeg 2010;172:3325-9.

3. Lysholm J, Wiklander J. Injuries in runners. Am J Sports Med 1987;15:168-71.

4. Clement DB, Taunton JE, Smart GW. Achilles tendinitis and peritendinitis: etiology and treatment. Am J Sports Med 1984;12:179-84.

5. Van Middelkoop M, Kolkman J, Van Ochten J, et al. Prevalence and incidence of lower extremity injuries in male marathon runners. Scand J Med Sci Sports 2008;18:140-4.

6. de Vos RJ, Weir A, van Schie HT, et al. Platelet-rich plasma injection for chronic Achilles tendinopathy: a randomized controlled trial. JAMA 2010;303:144-9.

7. Paavola M, Orava S, Leppilahti J, et al. Chronic Achilles tendon overuse injury: complications after surgical treatment. An analysis of 432 consecutive patients. Am J Sports Med 2000;28:77-82.

8. Schepsis AA, Jones $\mathrm{H}$, Haas AL. Achilles tendon disorders in athletes. Am J Sports Med 2002;30:287-305.

9. Lamberts H, Wood M, Hofmans-Okkes I. The International Classification of Primary Care. Oxford: Oxford University press 1987.

10. Walker-Bone K, Palmer KT, Reading I, et al. Prevalence and impact of musculoskeletal disorders of the upper limb in the general population. Arthritis Rheum 2004;51:642-51.

11. Gaida JE, Ashe MC, Bass SL, et al. Is adiposity an under-recognized risk factor for tendinopathy? A systematic review. Arthritis Rheum 2009;61:840-9.

12. Picavet HS, Schouten JS. Musculoskeletal pain in the Netherlands: prevalences, consequences and risk groups, the DMC(3)-study. Pain 2003;102:167-78.

13. Cook JL, Khan KM, Purdam C. Achilles tendinopathy. Man Ther 2002; 7:121-30. 